\title{
Sombrillas y operaciones en la construcción científica del
} mundo*

\author{
Umbrellas and Operations in the Scientific Construction of the World
}

Alfonso Arroyo-Santos ${ }^{\dagger}$

\begin{abstract}
Resumen
En este trabajo se argumenta que muchas de las controversias conceptuales en biología evolutiva se deben al uso de diversas definiciones operativas. Al no ser consistente el conjunto de operacionalizaciones, entonces hay muchas maneras de entender, en la práctica, a qué refiere, y cómo debe investigarse, cierto concepto. En este trabajo se presenta un desarrollo formal para estudiar el alcance del operacionalismo y de ahí se concluye que los conceptos controversiales son en realidad variables sombrilla, esto es, que un mismo término pueda referir a procesos, fenómenos o entidades distintas pero que se encuentran vinculadas por un gran presupuesto ontológico detrás de la operacionalización. Si esto es correcto, queda que muchas de las controversias en el campo se deben, en parte, a la operacionalización de los objetos de estudio.
\end{abstract}

Palabras clave: definición operativa - controversias - biología evolutiva - radiación adaptativa

\begin{abstract}
This paper argues that many of the conceptual controversies in evolutionary biology are due to the use of different operational definitions. Since the set of operations is not consistent, then there are many ways to understand, in practice, how concepts should be investigated. In this paper we introduce a formal analysis to study the scope of operationalism and we conclude that the controversial concepts are in fact umbrella variables, that is, a single term can be referring to different processes, phenomena or entities linked by a large ontological commitment driving operationalism. If this is correct, then many of the controversies in evolutionary biology are due, in part, to the operationalization of the objects of study.
\end{abstract}

Keywords: operative definition - controversies - evolutionary biology - adaptive radiation

* Recibido: 3 de Julio de 2016. Aceptado con revisiones: 13 de Agosto de 2016.

† Centro de Información Geoprospectiva y Facultad de Filosofia y Letras, Universidad Nacional Autónoma de México. Para contactar al autor, por favor, escribir a: agripas@gmail.com.

* Agradezco a Pablo Lorenzano, a la audiencia que participó en AIFIBI y a un revisor anónimo, cuyos comentarios mejoraron en gran medida este texto.

Metatheoria 8(1)(2017): 107-116. ISSN 1853-2322.

(c) Editorial de la Universidad Nacional de Tres de Febrero. Publicado en la República Argentina. 


\section{Introducción}

Existen al presente grandes controversias sobre el significado de diversos conceptos en biología. Por citar sólo algunas, queda en duda qué es una especie, a qué refiere el nicho ecológico, qué es la radiación adaptativa, qué es exactamente un gen, qué es la información biológica (Beurton et al. 2000, Griffiths 2001, Moss 2003, Olson \& Arroyo-Santos 2009). Algunos de estos conceptos han sido caracterizados como metafóricos y, por tanto, de ser imprecisos para el desarrollo de la ciencia. En este trabajo se sostiene que muchas de estas controversias tienen su origen en el estatus operativo de los conceptos involucrados. Esto es, muchos de los conceptos problemáticos están construidos sobre un conjunto de definiciones operativas cuya función es la de servir de base para la investigación empírica.

En este sentido, el concepto viene vinculado a un conjunto de maneras en que puede ser pensado, cuantificado, concretizado, inferido de los resultados empíricos. Dado que el concepto recoge muchas maneras diferentes en que puede ser utilizado en la práctica, es difícil encontrar un consenso que permita caracterizar el concepto de manera unívoca y es por ello, que distintos grupos de investigación entran en controversia al apoyar definiciones distintas Para dar cuenta de la idea que en un concepto problemático quedan vinculadas muchas definiciones operativas, se dirá que tales conceptos funcionan como "variables sombrilla". La idea central es que la variable sombrilla recoge muchas maneras en las que un concepto puede ser investigado y, por tanto, vinculado a entidades, fenómenos o procesos distintos.

Hablar de operacionalismo puede sonar arcaico toda vez que el proyecto con tal nombre impulsado por Bridgman en la década de 1920, fue cuestionado hace muchos años por los filósofos de la ciencia. Pero si bien el análisis filosófico de las definiciones operativas fue crítico, su uso en la ciencia contemporánea es constante lo que ha llevado a un grupo de filósofos a construir un "nuevo" operacionalismo, alejado de las críticas a Bridgman, y fiel a la práctica científica (Chang 2004, 2011, Feest 2005, 2010, 2011a, 2011b). En los nuevos análisis, lo que interesa saber es cómo se utilizan los conceptos en la práctica, y entender de qué manera se les va vinculando a objetos de investigación científica. Es por ello que, si el nuevo operacionalismo es una explicación correcta de la construcción de conceptos biológicos, queda entonces que la mayoría de los conceptos biológicos en controversia al presente vendrían a ser en esencia, definiciones operativas, en tanto que se toman como conceptos ya definidos cuando se trata de conceptos en vías de construcción, y, además, se trata de conceptos para los que todavía no existe suficiente evidencia empírica que permita una definición unívoca.

Si el análisis que se presenta es correcto, entonces es posible defender que muchas de las controversias en filosofía de la ciencia sobre el estatus de ciertos conceptos (gen, radiación adaptativa, especie...) se debe a un análisis incorrecto de lo que son los conceptos. Las controversias conceptuales en biología sólo tienen sentido bajo la idea de que los términos científicos son estables cuando en realidad se trata de etiquetas que agrupan importantes vacíos de conocimiento, y grandes maneras en que se atacan dichos vacíos en la práctica. El argumento se desarrollará de la siguiente manera. En la primera parte se discutirá qué es una definición operativa. En la segunda parte se presentará brevemente el caso de la radiación adaptativa, uno de los conceptos fundamentales de la biología evolutiva contemporánea que actualmente presenta problemas conceptuales importantes. En base a trabajo propio y de otros investigadores, se mostrará cómo la radiación adaptativa ha sido operacionalizada extensamente con el propósito de "identificar los mecanismos causales detrás del concepto" (Glor 2010). Sin embargo, el resultado ha sido una profusión de maneras de entender el fenómeno. En la tercera parte se discutirán los problemas conceptuales en torno a la radiación en el marco de las definiciones operativas, y finalmente, en la cuarta sección se discutirán los alcances de la propuesta. 


\section{2. ¿Qué es una definición operativa?}

En 1927 Brigman escribe:

¿Qué entendemos por la longitud de un objeto? Evidentemente, sabemos lo que entendemos por longitud si podemos decir cuál es la longitud de cualquier objeto, y para el físico, nada más es necesario. Para encontrar la longitud de un objeto, tenemos que realizar ciertas operaciones físicas. El concepto de longitud, por tanto, se fija cuando las operaciones para medir la longitud son establecidas: es decir, el concepto de longitud implica tanto y nada más, que el conjunto de operaciones por las que se determina la longitud. En general, se entiende por cualquier concepto nada más que un conjunto de operaciones; el concepto es sinónimo con su conjunto de operaciones correspondiente (Bridgman 1927, p. 5).

Tras una recepción positiva por parte de los miembros del Círculo de Viena durante la década de 1930, quienes vieron en el trabajo de Bridgman apoyo para sus propias ideas filosóficas, en particular, la tesis del verificacionismo, el operacionalismo fue descartado por varias razones, la principal, por haber sido entendido como una teoría del significado en la que los conceptos adquirían sentido a través de su manipulación experimental, tesis que enfrentaría muchos problemas (Chang 2009).

Desde hace algunos años se ha desarrollado un nuevo tipo de operacionalismo enmarcado dentro de una corriente filosófica llamada "epistemología histórica" (Feest \& Sturm 2011). Los seguidores del nuevo operacionalismo afirman que su trabajo está inspirado en aquellos elementos positivos que aportó el trabajo de Bridgman, pero que su interés no es defender que el conjunto de definiciones operativas confiere de significado al concepto sino únicamente, les interesa comprender cómo se desarrollan las operaciones para intervenir en el mundo. En palabras de Uljana Feest, su interés es: "cómo dar cuenta de los procesos dinámicos mediante los cuales, los objetos de investigación son conceptualizados e investigados" (Feest 2011a, p. 392).

La idea básica dentro del operacionalismo adoptado por Feest es que, para hacer el trabajo empírico que permitirá la investigación de un nuevo fenómeno, se debe comenzar con una idea preliminar que sirva de guía para la investigación empírica. Esto significa que hay que dar por sentado ciertos aspectos del nuevo objeto que servirán como punto de partida para la investigación (Feest 2011a, p. 392). De esta manera, las definiciones operativas constituyen el punto de partida y la dirección de las intervenciones experimentales que ayudarán a investigar el nuevo objeto. Pero ¿cómo se sabe qué tomar por sentado? Según Feest, la base viene de los supuestos conceptuales anteriores al nuevo objeto de investigación, heredados en la forma de "paradigmas experimentales". Los paradigmas experimentales son "ejemplares compartidos en el contexto de la investigación empírica que proveen de las condiciones paradigmáticas de aplicación” (Feest 2011a, p. 403, Feest \& Sturm 2011). En este sentido, el paradigma dicta cómo interpretar los resultados experimentales, y cómo los resultados permiten la construcción conceptual de un nuevo objeto.

Es importante hacer notar que, en el fondo, las ideas expuestas por Feest no difieren de aquello que propuso Bridgman en su tiempo. La razón principal se debe a que los seguidores del nuevo operacionalismo asumen que muchas de las críticas que recibió el trabajo Bridgman se debieron, por una parte, a reconstrucciones hechas por otros filósofos quienes quisieron ver en el operacionalismo la defensa de un empirismo radical, lo que implica que no se criticó a Brigman directamente, o bien, porque Bridgman nunca fue claro en la presentación de sus tesis lo que llevó a muchas incomprensiones (Chang 2009).

El nuevo operacionalismo pretende rescatar lo que entienden, era la verdadera motivación de Bridgman, a saber, construir una teoría pragmática sobre cómo se construyen o descubren los objetos científicos (Feest 2011, 2011a, Chang 2009). Dado el naturalismo de Feest, siempre hay, dentro de un paradigma científico, una idea de cómo investigar el objeto desconocido. Si esto es correcto, entonces las definiciones operativas introducen normas que guían la práctica científica. Esta noción vincula la definición operativa y el objeto de estudio en una relación dinámica que ayudará a definir el objeto desconocido de acuerdo con la "máxima metodológica de operacionalismo" que exige "una explicación de los supuestos conceptuales implícitos en un entorno de investigación determinado, con lo cual es 
posible la evaluación crítica y permite detectar fuentes de error" (Feest 2011a, pp. 403-404). Por ejemplo, en el estudio de caso de Feest, la memoria a corto plazo, "siempre" ha habido una comprensión tosca de lo que es la memoria, y por tanto "los conceptos relevantes están ligados a maneras en que la evidencia se detecta en la forma de un conocimiento previo; es decir, a paradigmas experimentales y procedimientos experimentales específicos" (Feest 2010, 2011a).

Para ilustrar su tesis, Feest analiza el caso de la memoria a corto plazo. Brevemente, las operaciones involucradas en estudiar un objeto llamado "memoria a corto plazo" comenzaron en la década de 1960 con los trabajos de Atkinson y Schiffrin. La base de dichos estudios es la cuantificación de la duración y la capacidad de la memoria, lo que se traduce en medir el tiempo al que refiere la memoria de corto plazo y qué tanta información se puede retener en este lapso. En ambos casos, los experimentos involucran pedir a un grupo de voluntarios recordar cierto número y tipo de objetos tras serles presentados a distintos intervalos de tiempo (Feest 2010, 2011a). Según el recuento ofrecido por Feest, los resultados experimentales han arrojado una gran cantidad de datos que, en los hechos, no permiten una definición unívoca de lo que es la memoria a corto plazo ya que "la pregunta de cómo caracterizar la memoria de corto plazo empíricamente, está atada a [distintas] cuestiones teóricas, metodológicas o incluso, terminológicas" (Feest 2011a, p. 396). Es importante notar que, al menos en el contexto de los estudios de la memoria a corto plazo "los paradigmas experimentales son bastante limitados" (Feest 2011a, p. 402), lo que permite que "a pesar de las cuestiones terminológicas y teóricas confusas, hay algo de acuerdo sobre la existencia de dos tipos de efectos empíricos: aquellos que están, y aquellos que no están, correlacionados con otras habilidades cognitivas como la inteligencia” (Feest 2011a, p. 397).

Aunque Feest es muy cauta con respecto al alcance epistemológico de su propuesta, y prefiere guardar silencio en si el nuevo operacionalismo implica cierto tipo de realismo, en el sentido de si las definiciones operativas conducen finalmente a la descripción de objetos de la naturaleza, sí considera que el nuevo operacionalismo ofrece una norma sobre una práctica científica adecuada (Feest 2011a, p. 403). Según Feest, el nuevo operacionalismo norma la manera en que los científicos deberían investigar objetos poco conocidos, no sólo al utilizar definiciones operativas sino al aclarar qué supuestos se encuentran detrás de cada definición. Idealmente, la operacionalización permitirá la individuación de los objetos, aunque esto no implique llegar a una definición unívoca, como muestra el ejemplo de la memoria de corto plazo, que, si bien tiene diferentes connotaciones, todas ellas dejan claro que se trata de un objeto vinculado a ciertas habilidades cognitivas.

Este trabajo pone en duda la tesis de Feest y demás seguidores del nuevo operacionalismo, de que las definiciones operativas suponen la introducción de una metodología de la investigación que permite ir conociendo nuevos objetos científicos. La clave para nosotros radica en la idea, compartida por todos los seguidores del nuevo operacionalismo, de que, a pesar de ciertas inconsistencias terminológicas, el operacionalismo permite la individuación de nuevos objetos. En nuestra opinión, si algo logra el operacionalismo es ampliar el conjunto de características, procesos y fenómenos vinculados a cierto objeto, en lugar de irlos acotando como se defiende en el recuento de Feest. Si nuestra tesis es correcta, entonces la operacionalización permite vincular diferentes fenómenos, procesos e incluso entidades, a un mismo concepto. Por esta razón, llamaremos a dicho concepto "variable sombrilla" con lo que queremos destacar que no se trata de un objeto científico único, sino de un conjunto de entidades diferentes. Para defender nuestra tesis, a continuación, se presentará el caso de la radiación adaptativa, uno de los conceptos básicos de la biología evolutiva contemporánea, que a pesar de contar con una larga historia de operacionalización, al presente su concepción es ambigua e incluso contradictoria.

Para analizar el estudio de caso, se propone un modelo en que las definiciones operativas, la investigación empírica y el mundo, configuran una relación entre funciones como se ilustra en la Figura 1. 
Figura 1

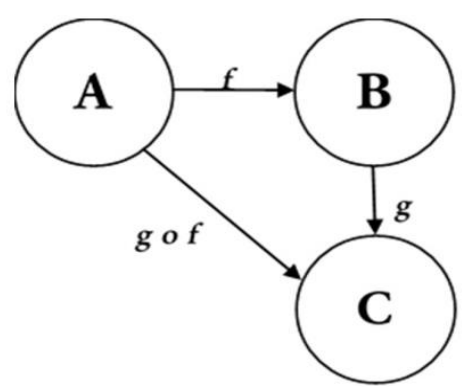

Representación diagramática entre definiciones operativas (A), los resultados de la investigación empírica que configurarán lo que se llamará objeto epistémico (B) y el mundo (C). f y g son funciones que van de $\mathrm{A}$ a $\mathrm{B}$ y de $\mathrm{B}$ a $\mathrm{C}$, respectivamente, y g o f (la función g de f), representa la relación que existiría entre la definición operativa y el mundo.

En este trabajo, la definición operativa es una función (f) que va de la cuantificación de ciertas variables (A) a ciertos conceptos que llamaremos, siguiendo lo que dicen los miembros del nuevo operacionalismo, objetos epistémicos (Feest 2011a, Chang 2011) (B). Con este vocabulario, lo que se quiere decir es que el objeto epistémico no es en realidad el concepto que da cuenta de la naturaleza, sino una entidad en la que se interviene operativamente con el propósito de elucidar el objeto científico representado por C. Hacer notar que $\mathrm{C}$, el objeto científico, es, por ejemplo, aquello que se entiende por memoria a corto plazo, o por radiación adaptativa, en tanto que B representa las maneras en que se conceptualizó temporalmente la memoria a corto plazo en el proceso de investigación.

\section{3. ¿Qué es la radiación adaptativa?}

La radiación adaptativa nació a finales del siglo XIX cuando el paleontólogo Henry F. Osborn acuñó el término para "[e]xpresar más claramente la idea de diferenciación de hábitats en varias direcciones a partir de un prototipo primitivo" (Osborn 1902, p. 353). Con esta terminología, Osborn quería separar su idea de radiación adaptativa de lo que entonces se llamaba evolución divergente, esto es, las diferencias que se dan en el tiempo entre especies que descienden de un ancestro común. Según Osborn, la radiación adaptativa es "[m]ás que la divergencia porque implica evolución en todas direcciones a partir de una forma central" (Osborn 1910, p. 78). Es importante subrayar que Osborn pensaba que "[e]sta idea de radiación, es una manera de interpretar e imaginar las relaciones entre la fauna viva y extinta" (Osborn 1910, p. 354). En resumen, la idea de radiación adaptativa fue introducida para cumplir con al menos, tres tareas principales: como una herramienta heurística para interpretar e imaginar las relaciones entre la fauna viva y extinta, como un término que pretendía aclarar problemas teóricos con la noción de divergencia, y como un descriptor de lo que Osborn consideraba una ley de la naturaleza.

Tras la introducción de Osborn, la idea de radiación adaptativa fue reinterpretada a lo largo de la primera mitad del siglo XX, sobre todo por el auge de lo se conoce como la Síntesis Moderna. Wright, por ejemplo, reformuló el concepto para adecuarlo a su paisaje adaptativo. Wright introdujo la idea de que las distintas subpoblaciones de una especie debían estar sujetas a diferentes presiones selectivas, lo que resulta en adaptaciones divergentes. Esta interpretación significó cambiar la tónica general del concepto, de describir cómo surgen nuevos taxones a partir de un ancestro común, a explicar cómo las presiones selectivas llevan a las poblaciones a nuevos picos adaptativos cuyo resultado es la radiación adaptativa de los organismos. (Wright 1931, 1932). Años más tarde, Simpson, uno de los padres de la síntesis moderna, terminó de cimentar la importancia del concepto al decir que la radiación adaptativa es "[m]ás o menos la divergencia simultánea de numerosas líneas de un mismo tipo ancestral adaptativo, a zonas adaptativas diferentes también divergentes" (Simpson 1953, p. 222). Aunque la descripción de Simpson está totalmente en la línea de Wright, Simpson desplazó el énfasis del 
concepto hacia la naturaleza adaptativa de la divergencia entre linajes. De esta manera, enfatizó la preocupación de la síntesis moderna de que el cambio evolutivo está impulsado principalmente por procesos de adaptación.

Si bien la conceptualización de Simpson quedaría como la definición canónica de radiación adaptativa, a partir de la segunda mitad del siglo XX, el auge de nuevas técnicas experimentales, sobre todo aquellas venidas del campo de la biología molecular, provocaría una explosión en la operacionalización y cuantificación del concepto. Actualmente se han cuantificado factores tales como la tasa de especiación, tasa de cambios morfológicos, o la distribución desigual de las especies en linajes evolutivos. Todo ello con el fin de aclarar aquello del "más o menos" de Simpson. Por ejemplo, Stanley (1979) cuantificó la tasa de especiación entre distintos procesos y concluyó que la radiación adaptativa se caracteriza por procesos de especiación muy rápidos, donde rápido refiere a un corte temporal elegido por Stanley. En otro caso, Guyer y Slowinski (1993) midieron la distribución filogenética de las especies para decir que la radiación adaptativa se caracteriza por la presencia de grupos con un número significativamente mayor de especies que su linaje más estrechamente relacionado, donde lo significativo, de nueva cuenta, fue un corte elegido por los autores. En cualquier caso, los esfuerzos por cuantificar la radiación adaptativa han dado como resultado una literatura enorme que en lugar de ayudar a aclarar qué es, ha ampliado su abanico de significados posibles ya que se han construido tantas interpretaciones como indicadores cuantitativos se han imaginado (Losos 2010, Glor 2010, Olson \& Arroyo Santos 2009).

El breve recuento histórico presentado tiene tres objetivos principales: 1) mostrar cómo la radiación adaptativa es un concepto con gran éxito empírico y conceptual en biología evolutiva, a pesar de no quedar claro qué es, o a qué parte de la naturaleza refiere. 2) Subrayar que, tras su introducción, la noción de radiación adaptativa se reformuló según distintas presiones teóricas, pero siempre ha mantenido la intuición original de muchas especies "que irradian" de un ancestro común. 3) Mostrar cómo la investigación contemporánea ha contribuido a ampliar el conjunto de fenómenos que se asociación a la radiación adaptativa mediante la cuantificación de diversos aspectos.

\section{Sombrillas y radiaciones}

De acuerdo con la tesis de este trabajo, los problemas conceptuales en torno a la radiación adaptativa tienen que ver con el conjunto de definiciones operativas que se han desarrollado con el fin de clarificar el concepto. En este sentido, "radiación adaptativa" es una variable sombrilla que agrupa distintas maneras de operacionalizar cierto fenómeno con el fin de intervenirlo, explorarlo, comprenderlo. A continuación, se desarrolla un ejemplo.

Retomando la figura 1, existe una función de A a B que vincula una definición operativa con un objeto epistémico. En este caso, el objeto epistémico es la variable paraguas "radiación adaptativa" y la definición operativa puede ser, por ejemplo, la distribución filogenética postulada por Guyer y Slowinski según se comentó en la sección anterior. Llamemos a la definición operativa DO(x), entonces queda que:

$\mathrm{DO}(\mathrm{x})=\operatorname{Pr}\left(\mathrm{X}_{\mathrm{t}}=x / \lambda\right)=e^{-\lambda t}\left(1-e^{-\lambda t}\right)^{x-1}$

Donde la probabilidad de que haya $\mathrm{X}$ número de especies en el tiempo $t$, viene dada por el cociente entre las tasas de especiación $\lambda$ y las tasas de extinción $\mu$. Para fines de ilustración, la ecuación 1 muestra el caso de la probabilidad de la distribución para el caso de una especie con $\mu=0$ (de allí que no sea representada en la ecuación) (Paradis 2011). Es muy importante observar que, según DO(x), la radiación adaptativa es un fenómeno asociado a la presencia de grupos filogenéticos con un número significativamente mayor de especies que el de su linaje más estrechamente relacionado. Es por esta razón que $\mathrm{DO}(\mathrm{x})$ cuantifica el número de especies en cierto horizonte temporal.

Comparar $\mathrm{DO}(\mathrm{x})$ con el caso de que la radiación adaptativa fuese asociada a la tasa de especiación según sugiere Stanley, en cuyo caso, la definición operativa, ahora llamada DO2(x) sería: 


$$
\mathrm{DO} 2(\mathrm{x})=\log \left[L\left(\sigma_{0}^{2}, \varphi\right)\right]=\sum_{1}^{n-1}\left[\left(-1 / 2 c_{i}^{2} / \varphi \cdot l_{i}+\sigma_{0}^{2}\right)-1 / 2 \log \left(\varphi \cdot l_{i}+\sigma_{0}^{2}\right)-\frac{1}{2} \log (2 \pi)\right]
$$

Donde: es la tasa de evolución fenotípica cuando el número de linajes co-occurentes es $0, \varphi$ es la pendiente de la relación entre la tasa y el número de linajes, $c_{i}$ es el score de contrastes filogenéticos (donde contraste refiere a la evaluación de las correlaciones entre filogenias como espurias o como genuinas), $l_{i}$ es la diversidad estimada del linaje en el nodo $i$ (Mahler et al. 2010).

Observar que la noción de radiación operativa que prefiere Stanley viene definida por procesos de especiación muy rápidos, y por ello, en DO2(x) se calcula la tasa de especiación.

Los ejemplos presentados en las ecuaciones (1) y (2) ilustran la diversidad en la operacionalización de la ración adaptativa, pero no olvidar que la lista de posibles definiciones operativas puede ser bastante larga dados los diversos intereses teóricos de los investigadores, según se comentó en la sección 3.

Según Feest y seguidores, las ecuaciones (1) y (2) son posibles gracias a una comprensión inicial del fenómeno que se quiere estudiar y los paradigmas experimentales vigentes para el caso de la biología evolutiva. En este caso, (1) y (2) operacionalizan la noción de que la radiación adaptativa está vinculada con tasas (rápidas) de especiación y la ausencia de balance filogenético. Si esto es así, entonces tenemos como resultado que el objeto A (ver figura 1) tendría entre sus miembros a $\mathrm{DO}(\mathrm{x})$ y $\mathrm{DO} 2(\mathrm{x})$, y que estos mapearían a según qué variables que conforman $\mathrm{B}$, o sea, el objeto epistémico radiación adaptativa, según se observa en la figura 2.

\section{Figura 2}

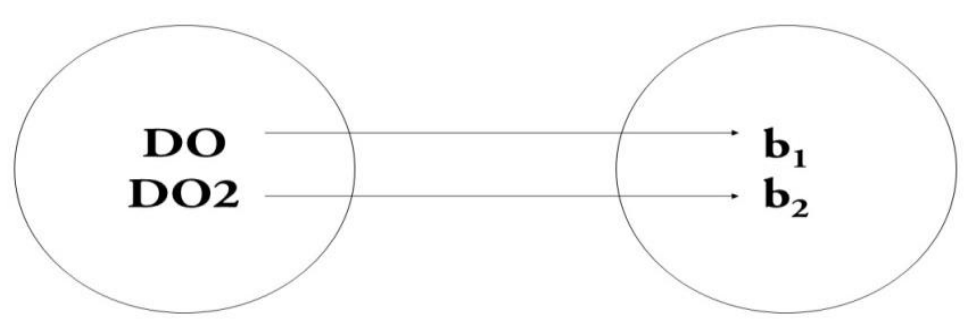

Función entre los elementos de A y B. DO y DO2 son dos definiciones operativas que forman parte del objeto A y a las que se les asigna un elemento del conjunto B.

Nótese que, a pesar de que $\mathrm{DO}(\mathrm{x})$ y $\mathrm{DO} 2(\mathrm{x})$ son funciones probabilísticas, se encuentran basadas en dos teorías sobre probabilidad diferentes. $\mathrm{DO}(\mathrm{x})$ es un ejemplo de probabilidad frecuentista en la que se asume que la probabilidad cuantifica un estado de la naturaleza mediante la repetición al infinito de un mismo experimento cuyos resultados constituyen una frecuencia cuyo límite es el valor de la probabilidad. En el ejemplo, $\mathrm{DO}(\mathrm{x})$ calcula la frecuencia con que teóricamente hubiera sido posible observar cierto número de especies en cierto tiempo. Por el contrario, DO2(x) es un ejemplo de probabilidad bayesiana y lo que se mide no es un estado de la naturaleza, sino un índice, llamado de verosimilitud, que calcula en una escala de 0 a 1, qué tanto apoyan los datos experimentales cierta hipótesis. En la ecuación (2) lo que se calcula es, dados ciertos datos empíricos (representados por $\sigma$ y $\varphi$ ) la medida en que se puede justificar, dado un modelo evolutivo (representado por $c_{i}$ y $l_{i}$ ), que hubo cierta tasa de especiación.

Aunque matemáticamente es posible vincular las teorías frecuentista y bayesiana, ontológicamente se trata de teorías muy diferentes: como se dijo, la teoría frecuentista, también llamada probabilidad objetiva, asume que la probabilidad calcula un estado del mundo, en tanto que el bayesianismo, también llamado probabilidad subjetiva, asume que la probabilidad calcula el vínculo entre datos e hipótesis. Aunque es posible discutir que si ciertos datos apoyan con fuerza cierta hipótesis es porque la hipótesis da cuenta fiel de un estado del mundo, el bayesianismo lo que evalúa es la hipótesis como un constructo teórico y no como una relación con la naturaleza; este segundo paso tendría que ser 
calculado por otros medios. Dadas las diferencias ontológicas entre frecuentismo y bayesianismo, proponemos que, en realidad, $\mathrm{DO}(\mathrm{x})$ y $\mathrm{DO} 2(\mathrm{x})$ no forman parte de un mismo conjunto, sino que cada función se vincula de diferente manera con el objeto epistémico B como se observa en la figura 3.

Figura 3

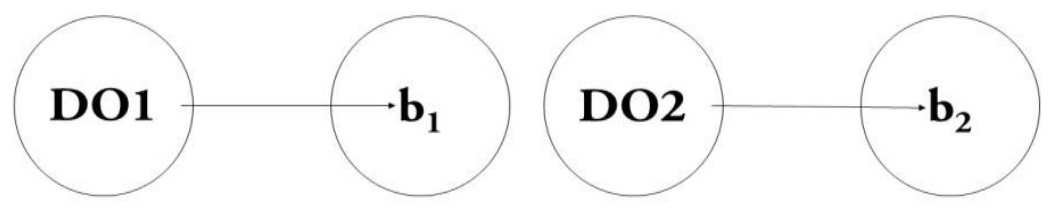

$A_{1}$ y $A_{2}$ representan dos objetos distintos que se vinculan con $B$.

¿Por qué $\mathrm{DO}(\mathrm{x})$ y $\mathrm{DO} 2(\mathrm{x})$ forman 2 conjuntos distintos y no son parte de un mismo conjunto que agrupe todas las definiciones operativas del objeto epistémico radiación adaptativa? El problema radica en las reglas que permiten saber si un elemento dado pertenece o no a un conjunto. Como se dijo, aunque matemáticamente es posible vincular las probabilidades frecuentista y bayesiana de manera que los resultados obtenidos por $\mathrm{DO}(\mathrm{x})$ y $\mathrm{DO} 2(\mathrm{x})$ pudiesen formar parte de un mismo conjunto, en la práctica, las diferencias ontológicas entre las dos teorías provocan que haya una definición operativa miembro de $A$, o sea que existe una $a_{1} \in A$ que se asignaría a un miembro de $B$, llamémoslo $b_{1}$, y que haya otra definición operativa de $A, a_{2} \in A$, que explícitamente dice que $b_{1}$ no puede ser miembro de B. Por ejemplo, los pinzones de Darwin, el ejemplo canónico de radiación adaptativa para cualquier libro de texto, no son casos de radiación para algunas definiciones operativas.

Pinto et al. (2008) construyeron su definición operativa sobre la base de una prueba de análisis de varianza, llamada ANOVA, tomando como base que el rasgo distintivo de la radiación adaptativa viene dado por la variación morfológica producto de la explotación de nuevos nichos ecológicos, justo la idea que sugirió Darwin para explicar la diversidad de los pinzones en las islas Galápagos. La prueba de ANOVA es una prueba estadística clásica de la probabilidad frecuentista que, para el caso del trabajo de Pinto y colaboradores, obtuvo como resultado contradecir uno de los supuestos principales de la explicación canónica de la radiación adaptativa, a saber, que el aislamiento geográfico, como es vivir en una isla separada del macizo continental, provoca casos de radiación adaptativa.

De este resultado se sigue que los problemas del concepto radiación adaptativa, y sólo en lo que se refiere a la construcción del objeto epistémico, se deben al uso de muchos objetos distintos $\left(\mathrm{a}_{1}, \mathrm{a}_{2}, \ldots\right.$, $\mathrm{a}_{\mathrm{n}}$ ) que mapean a un mismo objeto epistémico. Quizá un defensor del nuevo operacionalismo pudiera decir que $a_{1}, a_{2}, \ldots$ en realidad mapean distintas facetas del objeto $B$, esto es mapean $a b_{1}, b_{2}, \ldots, b_{n}$, y que ello da cuenta de la complejidad de B. Esto supondría que B requiere de distintas aproximaciones experimentales para ser aprehendido. Este resultado sería compatible con el pluralismo metodológico defendido por Feest. Sin embargo, este resultado no permite defender la tesis de que el operacionalismo permite la individuación del objeto en estudio, esto es, separar a la memoria de corto plazo de otros tipos de memoria, o separar a la radiación adaptativa de otros tipos de divergencia evolutiva. El nuevo operacionalismo asume que existe un conjunto pequeño de funciones que dan cuenta de cierto objeto epistémico y que el número de operaciones está restringido por los paradigmas experimentales. También asumen que existe una norma para la investigación por medio de operacionalizaciones, que permite la crítica de las funciones utilizadas, lo que, entendemos, permitiría mantener el número de funciones dentro de un rango pequeño, exhaustivo (todas las funciones dan cuenta de B) y coherente (no hay contradicción entre los miembros de los distintos conjuntos). Sin embargo, como se muestra para el caso de la radiación adaptativa, tal no ha sido el caso.

De acuerdo con la tesis de este trabajo, los problemas conceptuales en torno a la radiación adaptativa tienen que ver con el conjunto de definiciones operativas que se han desarrollado con el fin 
de clarificar el concepto. En este sentido, "radiación adaptativa" es una variable sombrilla que agrupa distintas maneras de operacionalizar cierto fenómeno con el fin de intervenirlo, explorarlo, comprenderlo.

\section{Conclusiones}

En este trabajo se ha tomado en serio el marco teórico del nuevo operacionalismo para decir que las definiciones operativas permiten aprender cosas nuevas del mundo por medio de la intervención experimental de ciertos objetos. Sin embargo, el análisis ha mostrado que al menos para el caso de la radiación adaptativa, el operacionalismo no permite la individualización de un objeto llamado radiación adaptativa, sino que más bien, la operacionalización ha permitido la construcción de muchos objetos vinculados a un mismo término. Pensamos que, si bien en este trabajo se ha mostrado el caso para la radiación adaptativa, los resultados pueden ser aplicables a otras controversias en biología. Por ejemplo, los casos de especie, nicho ecológico o información biológica. En todos estos casos, las controversias surgen porque fracasa la pretensión de que el concepto refiera unívocamente a un tipo de objeto, incluso si dicho objeto presenta controversias terminológicas o teóricas. Este punto es muy importante porque Feest presenta un recuento plural y relativista al aceptar 1) que el nuevo operacionalismo no pretende ser una teoría del significado, pero sí una teoría que explica cómo se estudian los objetos científicos en la práctica, y 2) que la operacionalización no necesariamente conduce al estudio de objetos de la naturaleza, pero sí que conduce a la individualización del objeto. En este trabajo se muestra que 2 no es correcto.

Nuestro análisis muestra que la operativización no necesariamente converge hacia un concepto consistente ya que los ejemplares paradigmáticos o el conocimiento previo no dan cuenta de una manera en que se ha de investigar ciertos fenómenos, sino más bien, dan cuenta de un gran presupuesto ontológico que puede ser investigado de muchas maneras. Proponemos que el operacionalismo construye variables sombrilla, esto es, que un mismo término pueda referir a procesos, fenómenos o entidades distintas pero que se encuentran vinculadas por ese gran presupuesto ontológico. Para el caso de la memoria de corto plazo, la experiencia de que hay cosas que se pueden recordar por lapsos cortos de tiempo y que dicha habilidad permite muchas tareas cognitivas, y para el caso de la radiación adaptativa, el presupuesto ontológico involucra diversidad evolutiva en periodos cortos de tiempo. Es nuestra tesis de que las variables sombrilla son la causa de muchas de las controversias conceptuales en biología evolutiva. Por ejemplo, el gen, la especie, o la información biológica, han sido operacionalizados de distintas maneras lo que no permite alcanzar el consenso que requiere la noción tradicional de concepto.

Bibliografía

Beurton, P.J., Falk, R. y H.J. Rheinberger (eds.) (2000), The Concept of the Gene in Development and Evolution: Historical and Epistemological Perspectives, Cambridge: Cambridge University Press.

Bridgman, P.W. (1927), The Logic of Modern Physics, New York: Macmillan.

Chang, H. (2004), Inventing Temperature: Measurement and Scientific Progress, New York: Oxford University Press.

Chang, H. (2009), "Operationalism", The Stanford Encyclopedia of Philosophy. http://plato.stanford.edu/archives/ fall2009/entries/operationalism/.

Chang, H. (2011), “The Persistence of Epistemic Objects Through Scientific Change”, Erkenntnis 75: 413-429.

Feest, U. (2005), "Operationism in Psychology-What the Debate is About, What the Debate Should Be About", Journal for the History of the Behavioral Sciences XLI: 131-150.

Feest, U. (2011a), “Remembering (Short-Term) Memory: Oscillations of an Epistemic Thing”, Erkenntnis 75: 391-411. 
Feest, U. (2011b), "What Exactly is Stabilized when Phenomena are Stabilized?", Synthese 182: 57-71.

Feest, U. y T. Sturm (2011), "What (Good) is Historical Epistemology? Editors Introduction”, Erkenntnis 75: 285-302.

Glor, R.E. (2010), "Phylogenetic Insights on Adaptive Radiation", Annual Review of Ecology and Evolutionary Systematics 41:251-270.

Griffiths, P.E. (2001), "Genetic Information: A Metaphor in Search of a Theory", Philosophy of Science 68: 394-412.

Guyer, C. y J.B. Slowinski (1993), "Adaptive Radiation and the Topology of Large Phylogenies”, Evolution 47: 253-263.

Losos, J.B. (2010), "Adaptive Radiation, Ecological Opportunity, and Evolutionary Determinism. American Society of Naturalists E.O. Wilson Award Address", The American Naturalist 175(6): 623-639.

Mahler, D.L, Revell, L.J., Glor, R.E. y J.B. Losos (2010), "Ecological Opportunity and the Rate of Morphological Evolution in the Diversification of Greater Antillean Anoles", Evolution 64(9): 2731-2745.

Moss, L. (2003), What Genes Can't Do, Cambridge: The MIT Press.

Olson, M.E. y A. Arroyo-Santos (2009), “Thinking in Continua: Beyond the 'Adaptive Radiation' Metaphor”, Bioessays 31: 1337-1346.

Osborn, H.F. (1902), "The Law of Adaptive Radiation”, American Naturalist 36: 353-363.

Osborn, H.F. (1910), "Paleontologic Evidence of Adaptive Radiation”, Popular Science Monthly 77: 77-81.

Paradis, E. (2011), "Shift in Diversification in Sister-Clade Comparisons: A More Powerful Test", Evolution 66: 288295.

Pinto, G., Mahler, D.L., Harmon, L.J. y J.B. Losos (2008), "Testing the Island Effect in Adaptive Radiation: Rates and Patterns of Morphological Diversification in Caribbean and Mainland Anolis Lizards", Proceedings of the Royal Society B: Biological Sciences 275: 2749-2757.

Rheinberger, H-J. (2000), "Gene Concepts: Fragments from the Perspective of Molecular Biology", en Beurton, P., Falk, R. y H.J. Rheinberger (eds.), The Concept of the Gene in Development and Evolution. Historical and Epistemological Perspectives, Cambridge: Cambridge University Press, pp. 219-239.

Simpson, G.G. (1953), The Major Features of Evolution, New York: Columbia University Press.

Stanley, S.M. (1979), Macroevolution: Pattern and Process, San Francisco: Freeman.

Wright, S. (1931), "Evolution in Mendelian Populations", Genetics 16: 97-159.

Wright, S. (1932), "The Roles of Mutation, Inbreeding, Crossbreeding, and Selection in Evolution", en Jones, D.F. (ed.), Proceedings of the Sixth International Congress of Genetics, Vol. 1, pp. 356-366. 